\title{
Comparison of Whole-Body PET/CT, Dedicated High-Resolution Head and Neck PET/CT, and Contrast-Enhanced CT in Preoperative Staging of Clinically Mo Squamous Cell Carcinoma of the Head and Neck
}

Rosana S. Rodrigues ${ }^{1}$, Fernando A. Bozza², Paul E. Christian ${ }^{3}$, John M. Hoffman ${ }^{4}$, Regan I. Butterfield ${ }^{3}$, Carl R. Christensen ${ }^{4}$, Marta Heilbrun ${ }^{4}$, Richard H. Wiggins, III $^{4}$, Jason P. Hunt ${ }^{5}$, Brandon G. Bentz ${ }^{5}$, Ying J. Hitchcock ${ }^{6}$, and Kathryn A. Morton ${ }^{4}$

${ }^{1}$ Biomaging-INBEB and Department of Radiology, Federal University of Rio de Janeiro, Rio de Janeiro, Brazil; ${ }^{2}$ Fundação Oswaldo Cruz and National Institute of Science and Technology in Structural Biology and Bioimaging-INBEB, Rio de Janeiro, Brazil;

${ }^{3}$ Huntsman Cancer Institute, University of Utah, Salt Lake City, Utah; ${ }^{4}$ Department of Radiology, University of Utah, Salt Lake City, Utah; ${ }^{5}$ Division of Otolaryngology-Head and Neck Surgery, Department of Surgery, University of Utah, Salt Lake City, Utah; and ${ }^{6}$ Department of Radiation Oncology, University of Utah, Salt Lake City, Utah

The purpose of this study was to compare optimized whole-body (WB) and dedicated high-resolution contrast-enhanced PET/CT protocols and contrast enhanced CT in the preoperative staging of primary squamous cell carcinoma of the head and neck. Methods: A total of 44 patients with clinically M0 squamous cell carcinoma of the head and neck underwent primary tumor resection and neck dissection within 6 wk of diagnostic imaging. Imaging consisted of a standard WB PET/CT protocol without intravenous contrast enhancement, followed by a high-resolution dedicated head and neck (HN) PET/CT protocol, which included diagnostic-quality contrast-enhanced CT (CECT). Imaging results were compared with histopathology. A 5-point scale was used to designate primary tumor localization and the presence of lymph node metastasis on a per-patient and per-level basis. For cervical nodes, receiver-operating-characteristic curves were generated to determine the differences in performance between the WB and HN PET/CT protocols and CECT. Sensitivity, specificity, positive and negative predictive values, and accuracy were calculated for primary tumor and cervical nodes. Results: No statistical difference was observed between WB and HN PET/CT protocols, both of which significantly outperformed CECT, in the evaluation of the primary tumor. The performance of the $\mathrm{HN}$ PET/CT protocol was superior to that of the WB PET/CT in the detection of cervical node metastases, achieving statistical significance on a per-level basis and approaching significance on a per-patient basis, with the greatest advantage in the detection of small positive lymph nodes $(<15 \mathrm{~mm})$. No significant difference

Received Jan. 11, 2009; revision accepted Apr. 8, 2009.

For correspondence or reprints contact: Kathryn A. Morton, Department of Radiology, University of Utah, 1A71 SOM, 50 N. Medical Dr., Salt

Lake City, UT 84132.

E-mail: kathryn.morton@hsc.utah.edu

COPYRIGHT () 2009 by the Society of Nuclear Medicine, Inc. was observed between the WB PET/CT protocol and CECT in nodal staging, either on a per-patient or on a per-level basis. Conclusion: The primary advantage of the dedicated HN PET/CT protocol over the WB protocol or CECT in the staging of head and neck cancer is in the detection of small lymph node metastases.

Key Words: positron emission tomography; computed tomography; head and neck cancer; squamous cell carcinoma; tumor staging

J Nucl Med 2009; 50:1205-1213

DOI: 10.2967/jnumed.109.062075

$\mathbf{T}$ he radiologic evaluation and staging of primary head and neck (HN) cancer have traditionally used both CT and MRI with low to moderate specificity in identifying both the primary tumor and the nodal involvement $(1,2)$. PET has become a well-established modality for the staging and therapeutic assessment of HN tumors (3-9). Several reports suggest that PET may be more sensitive and specific than MRI or CT in the nodal staging and characterization of the primary tumor (10). However, considerable debate remains as to whether the performance of any imaging modality is sufficient to supplant surgical or pathologic staging in $\mathrm{HN}$ tumors $(10,11)$.

The development of PET/CT, either with off-line fusion or with dedicated dual-modality scanners, combines tumoral metabolic assessment with high-resolution structural and anatomic information $(1,12)$. PET/CT is of value in charac- 
terizing the primary lesion, in staging nodes, and in identifying distant metastasis, with evidence of an effect in the management and outcome (13-17).

Acquisition and processing protocols may affect the performance of PET/CT. An increasing number of centers recognize the added value of obtaining a diagnostic-quality CT scan for PET/CT, and at least 1 previous report demonstrates better performance for a high-resolution dedicated HN PET scanner for the evaluation of patients with suspected $\mathrm{HN}$ cancer (18). However, there is no consensus regarding the best PET/CT protocol for maximizing lesion detectability and image quality for $\mathrm{HN}$ malignancies.

The overall goal of this project was to optimize the protocol for PET/CT for staging primary HN cancer. The specific aims of this study were to compare the performance of a high-resolution dedicated HN PET/CT scan using a diagnostic-quality contrast-enhanced CT (CECT), an optimized whole-body (WB) PET/CT scan, and a diagnosticquality CECT scan in staging clinically M0 squamous cell carcinoma (SCCA) of the head and neck.

\section{MATERIALS AND METHODS}

\section{Patient Population}

The study was approved by the institutional review board at the University of Utah. From a database of patients who underwent PET/CT between January 2005 and July 2007, the records of 236 consecutive patients referred for PET/CT for HN cancer were reviewed. Patients with SCCA of the head and neck (either in a primary site or in a nodal metastasis) who were considered potential candidates for curative surgery were identified. Exclusion criteria included HN malignancies other than SCCA, a delay in surgery longer than $6 \mathrm{wk}$ from the time of the PET/CT scan, the presence of known or suspected metastatic disease at the time of diagnosis, and inability of the patient to undergo both the WB and the HN PET/CT protocol, which included the intravenous administration of iodinated contrast. Patients were also excluded if they had received chemotherapy or radiotherapy during the 6 mo before PET/CT or during the interval between PET/CT and surgery. A total of 44 patients met the inclusion criteria.

\section{PET/CT Protocol}

After patients had fasted for $6 \mathrm{~h}$, their serum blood glucose level was measured and found to be less than $200 \mathrm{mg} / \mathrm{dL}$ in all cases. ${ }^{18} \mathrm{~F}-$ FDG (555 MBq [15 mCi]) was injected intravenously 90 min before the start of the first PET/CT scan. During the uptake interval, patients rested comfortably in a recliner at $75^{\circ} \mathrm{F}$, with head support, and were encouraged to minimize physical activity, talking, and chewing.

All patients were imaged on a commercial combined 16-slice PET/CT scanner (Biograph 16; Siemens). Two sequential imageacquisition protocols were performed for each patient. At $90 \mathrm{~min}$ after injection, a WB PET/CT acquisition protocol was first performed from the mid-forehead to the mid-thighs. For this examination, CT was performed using a body-imaging protocol with diagnostic exposure parameters but without the use of intravenous iodinated or oral contrast media. For the WB PET/CT protocol, patients were imaged in the standard PET/CT bed (cradle) using the built-in head holder, with their arms down at their sides. After the WB protocol, patients were allowed a 15-min break while the cradle was changed to a flat bed. Patients were repositioned with their chin extended and their head taped into position in a head holder, again with their arms down at their sides. A high-resolution HN PET/CT acquisition was started at $150 \mathrm{~min}$ after an injection of ${ }^{18} \mathrm{~F}-\mathrm{FDG}$. The CT portion of the HN PET/CT protocol was performed according to a HN soft-tissue protocol, using an intravenous bolus of $100-\mathrm{mL}$ iohexol (Omnipaque 300; GE Healthcare) iodinated contrast $1 \mathrm{~min}$ before the $\mathrm{CT}$ acquisition. Imaging acquisition and processing parameters for the WB and HN PET/CT protocols are shown in Table 1.

[Table 1]

\section{Surgery and Histopathology}

All 44 patients underwent primary tumor resection and unilateral or bilateral neck dissection (with en bloc removal of lymph nodes) with curative intent within 6 wk of the PET/CT scans. One of 3 head and neck surgeons at the University of Utah Huntsman Cancer Hospital performed each surgery. The gold standard for the presence or absence of tumor was histopathology, confirmed by light microscopy of frozen or formalin-fixed sections.

\section{Image Interpretation}

CT and PET/CT images were evaluated on a commercially available computer workstation (ViewSonic, eSoft version 4.0;

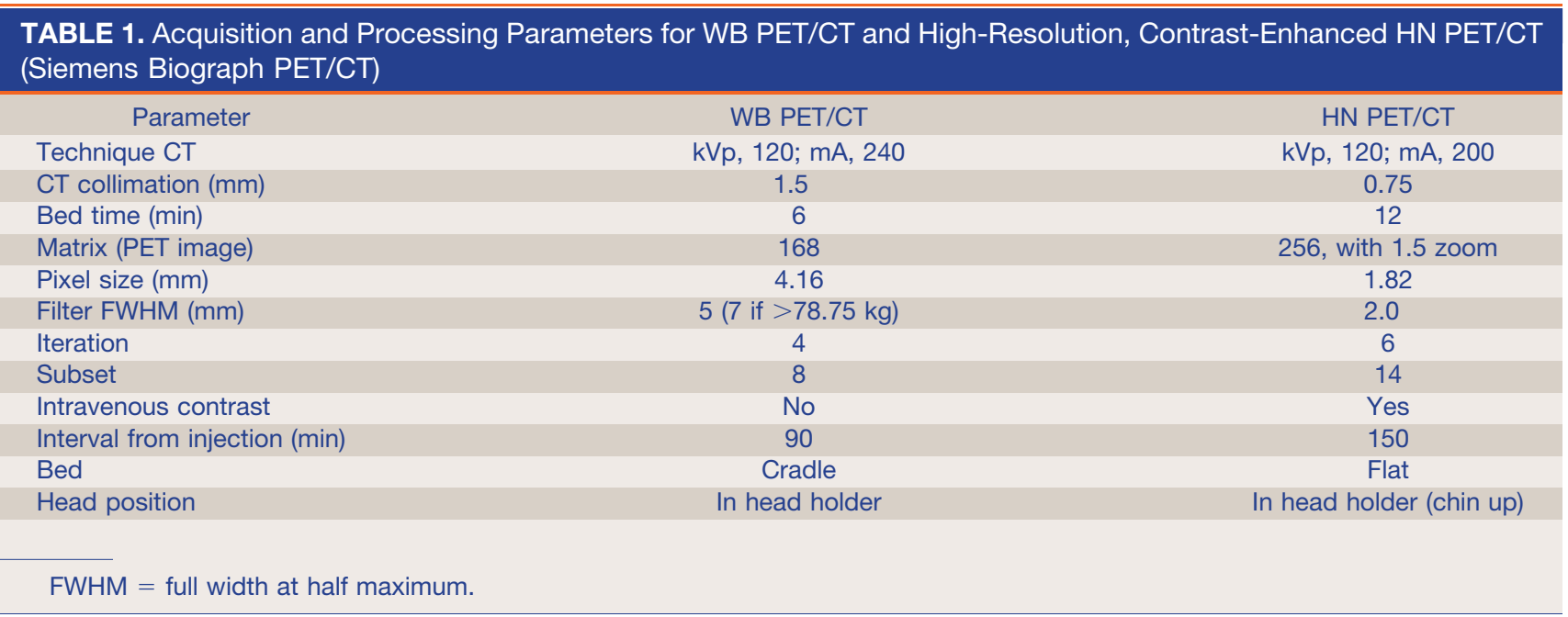


Siemens). The radiologists, without knowledge of the clinical data (including the site of the primary tumor), surgical or pathologic results, or previous imaging studies, independently reviewed the high-resolution CECT and the WB and HN PET/CT scans.

The primary tumor was evaluated by size, metabolic activity, and evidence for infiltration into adjacent structures. Metabolic activity was defined by a maximum standardized uptake value (SUVmax) and was corrected for body weight. A 5-point scale (1, definitely benign; 2 , probably benign; 3 , equivocal; 4 , probably malignant; and 5 , definitely malignant) was first used to designate primary tumor identification and infiltration into adjacent structures. A positive examination was defined as a score of 3-5, and a negative examination was defined as a score of 1-2.

The CECT scans were read independently of the WB and HN $\mathrm{PET} / \mathrm{CT}$ scans. In interpreting the CT scans, an overall receiveroperating-characteristic (ROC) score (1-5) was applied, as above. In addition, individual CT criteria were evaluated independently. One criterion was nodal size. Nodes were considered enlarged if they were greater than $10 \mathrm{~mm}$ in maximum short-axis diameter, except for retrophagyngeal nodes (enlarged if $>8 \mathrm{~mm}$ ) and jugulodigastric nodes (enlarged if $>15 \mathrm{~mm}$ ). Other criteria independently scored as suspected malignancy, regardless of nodal size, included the enhancement of lymph nodes by intravenous iodinated contrast (visually appreciable attenuation greater than adjacent muscle), the presence of necrosis, the absence of a normal fatty hila, a rounded (rather than oval) configuration (except for jugulodigastric nodes), indistinct nodal capsular margins, and asymmetry in size or number. If no enlarged or otherwise suspicious nodes were identified in a given level by CT, an ROC value of 1 (definitely normal) was assigned to that level.

By PET/CT criteria, lymph nodes considered suggestive of malignancy demonstrated visually appreciable metabolic activity above that of normal muscle or asymmetric metabolic activity greater than that of normal-appearing lymph nodes in the same level in the contralateral neck. For each lymph node level, an ROC score was assigned that represented the score of the most metabolically active node within that level. The SUVmax and size of that node were recorded. For nodal levels in which no metabolically active nodes were present or in which all nodes were less than $4 \mathrm{~mm}$ in diameter, a score of 1 (definitely abnormal) was assigned for those levels.

In addition to nodal scores on a per-level basis, a per-patient score for nodal metastases was defined for both PET/CT protocols and for CECT based on the highest single score for a nodal level for each patient. Discordant scores between the 2 radiologists were reviewed jointly and resolved by consensus.

\section{Statistical Analysis}

For the identification of the primary tumor, $\chi^{2}$ analysis was used to compare the performance of the WB and HN PET/CT protocols with that of the CECT protocol. A true-positive result was defined as correctly identifying the primary site of the tumor. A false-positive finding was defined as significantly misidentifying the site of the tumor or identifying a tumor at a site in which none was subsequently found. A $P$ value of less than 0.05 was defined as representing a statistically significant difference.

For nodal disease, ROC curves were generated to determine the differences in performance between the HN and WB PET/CT and the CECT protocols as defined by the 5-point scale, both on a perlevel basis and on a per-patient basis. Nodal levels for which pathologic correlation was available were analyzed. Comparison of the areas under the curve was made between the modalities using $z$ statistics. A statistically significant difference between modalities was defined as a $P$ value of less than 0.05 . Sensitivity, specificity, and positive and negative predictive values were also calculated, with a positive score defined as an ROC score of 3, 4, or 5 and a negative score as 1 or 2 .

\section{RESULTS}

A total of 44 patients met the inclusion criteria. The median interval between PET/CT and surgery was $19 \mathrm{~d}$ (range, 1$42 \mathrm{~d}$ ). The median patient age was $65 \mathrm{y}$ (range, 26-97 y). The patients included 35 men $(80 \%)$ and 9 women $(20 \%)$.

\section{Primary Tumor}

Among the 44 patients with SCCA, 34 were newly diagnosed and 10 had recurrence of a previously treated tumor (with no preceding treatment for at least $6 \mathrm{mo}$ ). In 38 of 44 patients (86\%), a histologically proven primary site of SCCA was observed. Six of 44 patients (14\%) presented with SCCA in enlarged lymph nodes without an identifiable primary tumor, despite rigorous panendoscopy. None of the imaging modalities (CT, WB PET/CT, or HN PET/CT) was successful in identifying an occult primary tumor in these 6 patients. The most frequent region for the primary tumor was the oropharynx (29 patients; 66\%), as shown in Table 2. A comparison of the different imaging modalities for detecting the primary tumor is summarized in Table 3.

Beam-hardening artifacts induced by dental amalgam prevented the CECT identification of the primary tumor in 14 of the 38 patients (37\%) who had a histologically proven primary tumor site. The primary tumor was correctly identified by CECT in 27 patients $(71 \%)$. In 2 patients (5\%), CECT suggested a primary tumor site that failed to be confirmed by histopathology. The sensitivity, specificity, accuracy, and positive and negative predictive values of contrast-enhanced CECT for the primary tumor were $71 \%, 67 \%, 70 \%, 93 \%$, and $27 \%$, respectively.

The WB PET/CT protocol correctly identified the site of the primary tumor in 35 of 38 patients $(92 \%)$. In the 3 cases $(8 \%)$ in which the primary tumor was not identified by the WB PET/CT protocol, the primary tumors were small (an in situ tumor, a skin tumor, and a 10-mm tumor). The HN PET/ CT protocol correctly identified the primary tumor site in 36 patients $(95 \%)$ and failed to identify the primary tumor in 2 patients (5\%). The 2 primary tumors not identified by the $\mathrm{HN}$ $\mathrm{PET} / \mathrm{CT}$ protocol were a small skin primary and an in situ tumor. The HN PET/CT protocol also misidentified a site of

\begin{tabular}{|lcc|}
\hline \hline TABLE 2. Distribution of Primary Tumor Location \\
\hline \multicolumn{1}{|c|}{ Primary tumor sites } & Number of patients & Total $(\%)$ \\
\hline Oropharyngeal & 29 & 66 \\
\hline Hypopharyngeal & 1 & 2 \\
\hline Laryngeal & 4 & 9 \\
\hline Skin & 4 & 9 \\
\hline Unknown primary site & 6 & 14 \\
\hline Total & 44 & 100 \\
\hline
\end{tabular}

Table 2]

[Table 3] 
TABLE 3. Detection of Primary Tumor by CECT, WB, and HN Protocols

\begin{tabular}{|c|c|c|c|c|c|}
\hline Imaging study & Sensitivity (\%) & Specificity (\%) & Accuracy (\%) & PPV (\%) & NPV (\%) \\
\hline CECT & 71 & 67 & 70 & 93 & 27 \\
\hline WB PET/CT protocol* & 92 & 100 & 93 & 100 & 67 \\
\hline HN PET/CT protocol ${ }^{\dagger}$ & 95 & 83 & 93 & 97 & 71 \\
\hline
\end{tabular}

periodontal disease as representing a possible primary tumor

[Fig. 1] in 1 patient (Fig. 1). The sensitivity, specificity, accuracy, and positive and negative predictive values of the WB PET/CT protocol for identifying the primary tumor were $92 \%, 100 \%$, $93 \%, 100 \%$, and $67 \%$, respectively, and they were $95 \%, 83 \%$, $93 \%$, 97\%, and $71 \%$, respectively, for the HN PET/CT protocol. There was no statistical difference in the detection of the primary tumor between $\mathrm{WB}$ and $\mathrm{HN}$ protocols; however, both the WB and HN protocols demonstrated significantly better performance than did CECT (Table 3) in identifying the primary site of the tumor $(P=0.0357$ and $P=0.0125$, respectively).

Histologic analysis of the surgical specimen demonstrated peritumoral infiltration in 31 of 38 of the primary tumors $(82 \%)$. Of these 31 , peritumoral infiltration was correctly identified in 21 of 31 cases $(68 \%)$ by both the CECT and the WB PET/CT protocols and in 22 of 31 cases (71\%) by the HN PET/CT protocol. A score of 5 (definitely abnormal) was assigned to 17 of 22 cases $(77 \%)$ of peritumoral infiltration by the HN PET/CT protocol but only 14 of $21(67 \%)$ by the WB PET/CT protocol. For identifying peritumoral infiltration of the primary tumor, the sensitivity, specificity, and positive and negative predictive values were $68 \%, 71 \%, 91 \%$, and $33 \%$, respectively, for the WB PET/CT protocol and 71\%, 57\%, 88\%, and $31 \%$, respectively, for the HN PET/CT protocol. These differences were not statistically significant.

The median SUVmax for the primary tumor was 13 (mean, 13; range, 3.70-30.6) by the WB PET/CT protocol and 14.7 (mean, 16.5; range, 5.30-47.3) by the HN PET/CT protocol. In 36 primary tumors, SUVmax was measured by both PET/ CT protocols. In all cases, the WB PET/CT protocol was

FIGURE 1. Site of infection or inflammation may mimic primary tumor by PET/CT. HN PET/CT shows falsepositive lesion due to peridontal abscess (solid arrow).

RGB Primary tumor was actually small skin lesion that was missed by all imaging modalities.

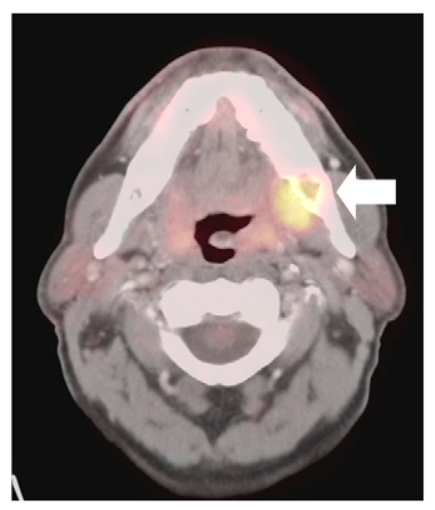

performed before the HN PET/CT protocol. In 32 of 36 cases (89\%), the SUVmax for the primary tumor increased significantly $(P=0.0119)$ between the WB PET/CT protocol (performed at 90 min after injection) and the HN PET/CT protocol (performed at $150 \mathrm{~min}$ ). As shown in Figure 2, between [Fig. 2] the WB and the HN PET/CT protocols, 24 cases (67\%) showed an increase in SUVmax by more than 20\%, 7 cases (19\%) showed an increase of 10\%-20\%, $1(3 \%)$ showed an increase of less than $10 \%$, and $4(11 \%)$ showed a decrease in SUVmax.

\section{Lymph Node Metastasis}

On a per-patient basis, lymph node metastases were identified histopathologically in 24 of 44 patients (55\%); 20 of 44 patients $(45 \%)$ had no nodal disease. The median number of lymph nodes analyzed histopathologically per level was 9 (mean, 11.68; range, 1-47). A total of 186 levels were evaluated histopathologically. Of these, 139 levels were tumor-free, and 47 levels were positive for tumor nodal involvement (prevalence, $25 \%$ on a per-level basis). The distribution of metastatic disease was similar among all nodal levels.

On a per-level basis, the sensitivity, specificity, accuracy, and positive and negative predictive values for the detection of nodal metastasis were $70 \%, 82 \%, 79 \%, 57 \%$, and $89 \%$, respectively, for the WB PET/CT protocol; $91 \%, 71 \%, 76 \%$, $51 \%$, and $96 \%$, respectively, for the HN PET/CT protocol; and $57 \%, 88 \%, 81 \%, 63 \%$, and $86 \%$, respectively, for the CECT protocol. Various CECT parameters were also individually evaluated in the identification of nodal tumor involvement and are shown in Table 4. The finding of [Table 4] heterogeneous enhancement was found to be the most accurate feature by CECT in identifying nodal involvement, with a sensitivity, specificity, accuracy, and positive and negative

FIGURE 2. Change in SUVmax from WB to HN protocol for primary tumor.

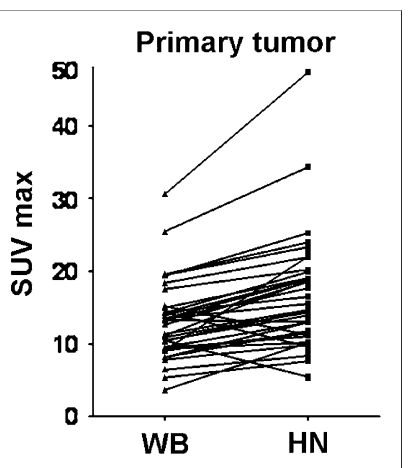


TABLE 4. Detection of Nodal Metastases per Level by CECT Parameters

\begin{tabular}{|c|c|c|c|c|c|}
\hline CECT feature & Sensitivity (\%) & Specificity (\%) & PPV (\%) & NPV (\%) & Accuracy (\%) \\
\hline Size (enlarged) & 63 & 89 & 66 & 88 & 79 \\
\hline Shape (round) & 83 & 66 & 45 & 92 & 73 \\
\hline Contrast enhancement (any) & 83 & 68 & 47 & 92 & 74 \\
\hline Heterogeneous enhancement & 63 & 96 & 84 & 89 & 83 \\
\hline Absence of fatty hilum & 97 & 21 & 29 & 95 & 51 \\
\hline Necrosis & 53 & 98 & 90 & 86 & 81 \\
\hline Extracapsular spread & 43 & 98 & 88 & 84 & 77 \\
\hline Asymmetry & 100 & 21 & 30 & 100 & 52 \\
\hline Overall CT appearance & 57 & 88 & 63 & 86 & 81 \\
\hline
\end{tabular}

predictive values for detection of nodal metastasis on a perlevel basis of $63 \%, 96 \%, 83 \%, 84 \%$, and $89 \%$.

By ROC analysis, the HN PET/CT protocol was superior to the WB PET/CT $(P=0.002)$ in the detection of nodal metastases on a per-level basis. On a per-patient basis, the HN PET/CT protocol showed a slightly better performance than did the WB PET/CT protocol, approaching significance $(P=$ $0.059)$. There was no significant difference between the WB $\mathrm{PET} / \mathrm{CT}$ protocol and the CECT protocol in the detection of nodal metastasis, either on a per-level $(P=0.10)$ or on a perpatient $(P=0.81)$ basis. The HN PET/CT protocol was significantly superior to CECT in the detection of nodal metastases on a per-level basis $(P<0.001)$ but not on a per-patient

[Fig. 3] basis $(P=0.15)$. The ROC curves are shown in Figure 3, and the [Table 5] statistical analysis of these curves is summarized in Table 5.

Metabolic activity (SUVmax) was compared in positive and negative nodes for both the WB and the HN PET/CT

[Fig. 4] protocols (Fig. 4). By the WB PET/CT protocol, the median SUVmax for positive nodes was 6.8 (mean, 9.1; range, 1.324.0), and the median SUVmax for negative nodes was 2.0 (mean, 2.8; range, 0.6-6.5). By the WB PET/CT protocol, no negative nodes with an SUVmax greater than 6.5 were seen. However, 18 of 35 (51\%) positive nodes had metabolic

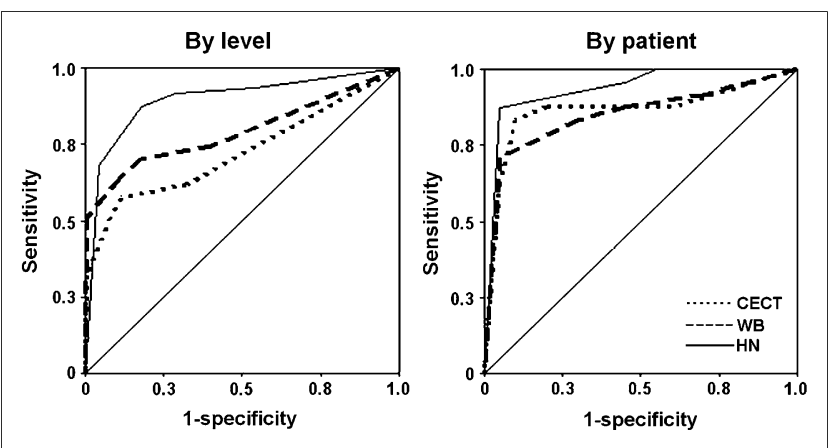

FIGURE 3. ROC analyses of CECT, WB PET/CT, and HN $\mathrm{PET} / \mathrm{CT}$ protocols in detection of nodal metastases by level and by patient. Dotted line = CECT; dashed line = WB; solid line $=\mathrm{HN}$. activity that fell within the range of negative nodes. By the HN PET/CT protocol, the median SUVmax for positive nodes was 8.1 (mean, 10.45; range, 1.1-31.0), and the median SUVmax for negative nodes was 2.6 (mean, 3.0; range, 0.4-9.6). By the HN PET/CT protocol, no negative nodes demonstrated an SUVmax greater than 9.6. However, 29 of $44(66 \%)$ positive nodes had SUVmax within the range of the negative nodes. An example of increased metabolic activity in both positive and negative nodes is illustrated in Figure 5. There was no significant difference in SUVmax in [Fig. 5] normal soft tissues, such as muscle or fat, between the HN and WB protocols.

For 82 lymph nodes, nodal SUVmax were measured at the same levels by both PET/CT protocols; 32 were histologically positive for tumor, and 50 were negative. The SUVmax for all nodes, both positive and negative, increased significantly $(P=0.007)$ from the WB PET/CT protocol (scan performed at 90 min after injection) to the HN PET/CT protocol (scan performed at 150 min after injection) (Fig. 6). [Fig. 6] However, there was no statistical difference in the change in SUVmax between the WB and the HN PET/CT protocols between positive and negative nodes (Table 6).

All of the tumor-containing lymph nodes in levels that proved falsely negative by PET/CT were small. The WB PET/CT protocol failed to identify 12 of 47 small positive lymph node levels where all false-negative nodes were less than $15 \mathrm{~mm}$ in short-axis diameter, and the HN PET/CT protocol failed to identify 3 of 47 positive lymph node levels where all of the false-negative nodes were less than $13 \mathrm{~mm}$ in short-axis diameter by histology. Representative PET/CT scans showing differences in the detection of small nodes between the WB and the HN PET/CT protocols are shown in Figure 7. There was no notation made, on the basis of the [Fig. 7] histologic reports, as to the degree to which the nodes were replaced by tumor.

\section{Distant Metastases}

Both the WB and the HN PET/CT protocols identified distant metastases in 1 of 44 patients. In this single patient, mediastinal nodal metastases were present. Three additional
[Table 6] 


\begin{tabular}{|c|c|c|}
\hline Parameter & By level & By patient \\
\hline \multicolumn{3}{|l|}{ Area under the curve } \\
\hline CECT & 0.722 & 0.863 \\
\hline WB protocol & 0.790 & 0.850 \\
\hline HN protocol & 0.896 & 0.936 \\
\hline \multicolumn{3}{|l|}{$95 \%$ confidence interval } \\
\hline CECT & $0.623-0.820$ & $0.741-0.984$ \\
\hline WB protocol & $0.699-0.881$ & $0.730-0.970$ \\
\hline HN protocol & $0.836-0.956$ & $0.860-1.000$ \\
\hline \multicolumn{3}{|c|}{ Comparison of CT vs. WB PET/CT } \\
\hline Difference between areas & 0.069 & 0.0125 \\
\hline Significance level & $P=0.099$ & $P=0.0811$ \\
\hline \multicolumn{3}{|c|}{ Comparison of CT vs. HN PET/CT } \\
\hline Difference between areas & 0.174 & 0.074 \\
\hline Significance level & $P<0.001$ & $P=0.152$ \\
\hline \multicolumn{3}{|c|}{ Comparison of WB vs HN PET/CT } \\
\hline Difference between areas & 0.106 & 0.086 \\
\hline Significance level & $P<0.002$ & $P=0.059$ \\
\hline
\end{tabular}

primary tumors, none clinically suspected, were detected by WB PET/CT among the 44 patients in this series. These included an adenocarcinoma of the colon, an inguinal lymph node melanoma metastasis, and lymphoma in axillary and inguinal lymph nodes.

\section{DISCUSSION}

There is no consensus regarding the best imaging approach for staging $\mathrm{HN}$ cancer. Reports of the performance of PET and PET/CT for staging HN cancer vary, with sensitivities from $70 \%$ to $100 \%$ and specificities from $78 \%$ to $100 \%$ $(17,18)$. Even greater variability exists in the reported performance of conventional imaging modalities (CT and MRI), with sensitivities ranging from $36 \%$ to $95 \%$ and specificities from $58 \%$ to $97 \%(18,19)$. Ultrasound shows early promise

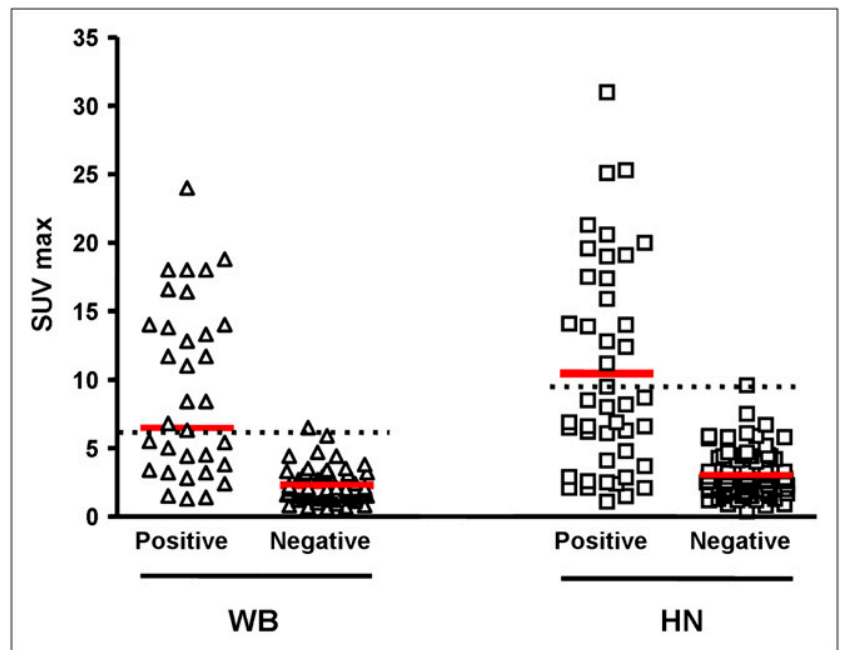

FIGURE 4. SUVmax in WB and HN protocols for positive and negative nodes. Dotted black line is placed just above highest SUVmax for negative nodes. Red line shows median of SUVmax for each group. for staging nodal disease, particularly when combined with fine-needle aspirate (20). Both technical factors and professional experience may contribute to performance variability among HN staging imaging modalities.

Yamamoto et al. report an improvement in small phantom lesion detection but not a statistically significant improvement in clinical lesion detection with a high-resolution, dedicated HN PET/CT protocol over a conventional PET/ CT protocol for nodal staging of $\mathrm{HN}$ cancer (18). The current study differs from that report in that all the current patients were clinically selected as being potentially resectable for cure (M0), had SCCA of the head and neck, and had histopathologic correlation within a short interval after the PET/CT scan. The current HN PET/CT protocol uses higherresolution acquisition and processing parameters, and comparison is made to a dedicated, diagnostic-quality CECT of the head and neck. Studies have shown progressive tumor ${ }^{18} \mathrm{~F}-\mathrm{FDG}$ uptake at intervals up to $3 \mathrm{~h}$ after injection (21-24). For the current study, 6-min bed positions were used for the WB PET/CT protocol, performed at $90 \mathrm{~min}$ after injection. The HN PET/CT protocol was performed at 150 min after injection and used 12-min bed positions. Thus, every attempt was made to optimize all imaging protocols.

FIGURE 5. PET/CT had difficulty in distinguishing reactive from malignant nodes. HN PET/CT protocol shows primary tumor (curved arrow), true-positive left level II node (open arrow), and false-positive right level II node (solid arrow). Both lymph nodes were similar in metabolic activity.

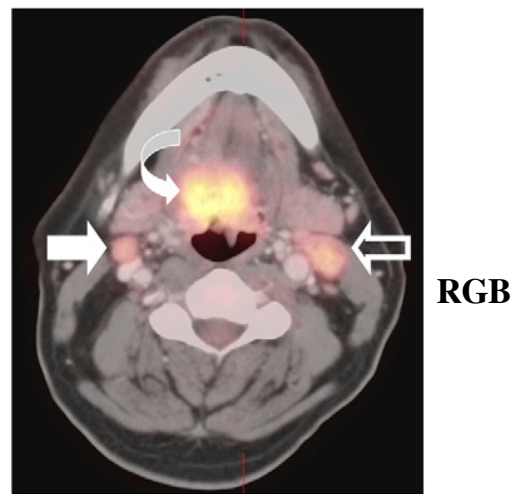



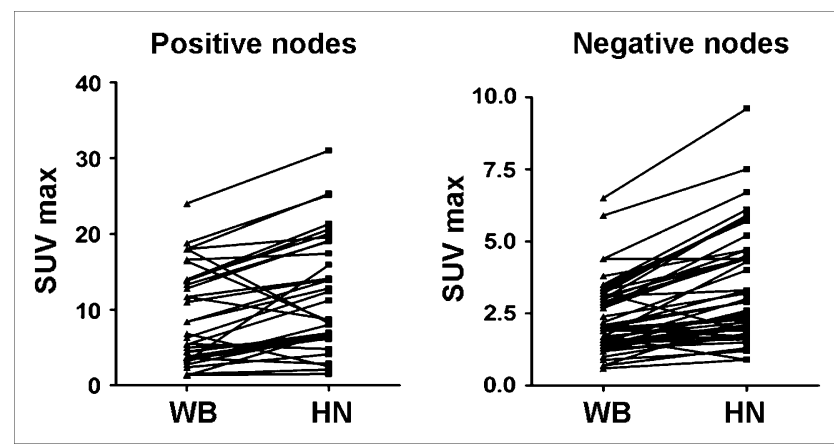

FIGURE 6. Change in SUVmax from WB PET/CT protocol (90 min after injection) to HN PET/CT protocol (150 min after injection) for positive and negative nodes.

Metallic beam-hardening artifacts produced by dental amalgam limited the CECT evaluation of the oral cavity and oral pharynx in $61 \%$ of patients. This limitation of the CECT protocol most likely contributed to the significantly better performance of both WB and HN PET/CT protocols when compared with CECT for identifying the primary tumor. These results are consistent with other reports (25). However, no difference in performance was found between WB and HN PET/CT protocols for identifying primary head and neck tumors. Tiny, in situ and skin primary lesions constituted false-negatives by both the HN and the WB PET/CT scan protocols. The HN PET/CT protocol erroneously identified a site of periodontal disease as representing a possible site of primary tumor.

There is debate regarding the value of PET or PET/CT in identifying the site of an occult $\mathrm{HN}$ primary cancer. Sensitivities of comparable reports range from $15 \%$ to $73 \%$ $(13,26-28)$. None of the imaging modalities in the current series identified the occult primary in the 6 patients who presented with nodal metastasis but no known primary tumor.

For nodal staging of the neck, the sensitivity and specificity were $70 \%$ and $82 \%$ for WB PET/CT, $91 \%$ and $71 \%$ for HN PET/CT, and 57\% and $88 \%$ for CECT. By ROC analysis, the HN PET/CT protocol demonstrated a significantly better performance than both the CECT and the WB PET/CT protocols in the detection of nodal metastases on a per-level basis $(P<0.001)$ and a near-significant improvement over WB PET/CT in the detection of nodal disease on a per-patient basis $(P=0.059)$. There was no significant difference between CECT and WB PET/CT in the detection of nodal metastases on a per-level $(P=0.10)$ or a perpatient $(P=0.81)$ basis or in the detection of nodal metastases on a per-patient basis $(P=0.15)$. Many studies have shown that PET/CT is superior to CT in the nodal staging of HN cancer. However, many of these studies compared PET to the WB CT scan obtained at the time of the PET scan and not to a dedicated CECT of the head and neck, possibly accounting for the relatively better performance of CECT in the current report.
TABLE 6. Change in SUVmax from WB to HN Protocol for Positive and Negative Nodes

$\begin{array}{lcc}\text { Change in SUVmax } & \text { Positive node* } & \text { Negative node } \\ \text { Increase } \geq 10 \% & 23(71.87) & 42(84.00) \\ \text { Increase } 0 \%-10 \% & 3(09.38) & 5(10.00) \\ \text { Decrease } & 6(18.75) & 3(06.00) \\ \text { Number of nodes } & 32(100.00) & 50(100.00)\end{array}$

*Data are number of nodes, with percentages in parentheses.

Others have shown a progressive rise in SUVmax over time, whereas metabolic activity within inflammatory lesions may plateau or drop (21-24). In the current study, however, neither magnitude of SUVmax nor an increase in SUVmax over time could reliably distinguish benign versus malignant lymph nodes by either the standard WB or the high-resolution HN PET/CT protocols. Metabolic activity (SUVmax) was significantly higher in positive than in negative nodes. No benign nodes were identified with an SUVmax greater than 9.6 for the HN protocol or greater than 6.5 for the WB protocol. However, $51 \%$ of all malignant nodes for the WB PET/CT protocol and $66 \%$ for the HN PET/CT protocol fell within the SUVmax range for benign nodes. A progressive increase in metabolic activity in both malignant and reactive nodes over time may have contributed to both the improved sensitivity and the slight reduction in specificity of the HN PET/CT protocol, when compared with the WB protocol. However, it could be argued that the identification of abnormal nodes on PET/CT, whether reactive or malignant, defines a vulnerable lymphatic region that must be addressed, either therapeutically or surgically.

Small lymph nodes accounted for most false-negative nodes by both the WB and the HN PET/CT protocol. In this regard, the higher-resolution HN PET/CT protocol had a significant advantage over the WB protocol and detected 4-fold more positive nodes $(<15 \mathrm{~mm})$ than did the WB protocol. The improvement in lesion detection with the $\mathrm{HN}$ PET/CT protocol, over the WB PET/CT protocol, could be attributed to a longer interval from injection to imaging and to finer sampling with smaller pixels $(1.82 \mathrm{~mm}$ for the $\mathrm{HN}$ protocol, which was supported by increasing data density by application of a 12-min bed position, as opposed to 4.16-mm pixels for the WB protocol). Smaller pixels reduce the partialvolume effect that reduces the visual intensity of small objects in addition to falsely lowering the SUVmax.

All of the patients in this series were clinically staged as M0 before PET/CT. Only 1 of 44 patients (2\%) proved to have distant metastatic disease (to mediastinal lymph nodes). This metastatic disease was visible both on the HN PET/CT protocol (which extends to the carina) and on the WB protocol. Other published series also report a low incidence of unsuspected metastatic disease detected by PET/CT in this population, in the range of $7 \%-15 \%(11,13)$.

Although the literature reports that PET/CT often alters management in $30 \%-35 \%$ of patients with $\mathrm{HN}$ cancer 


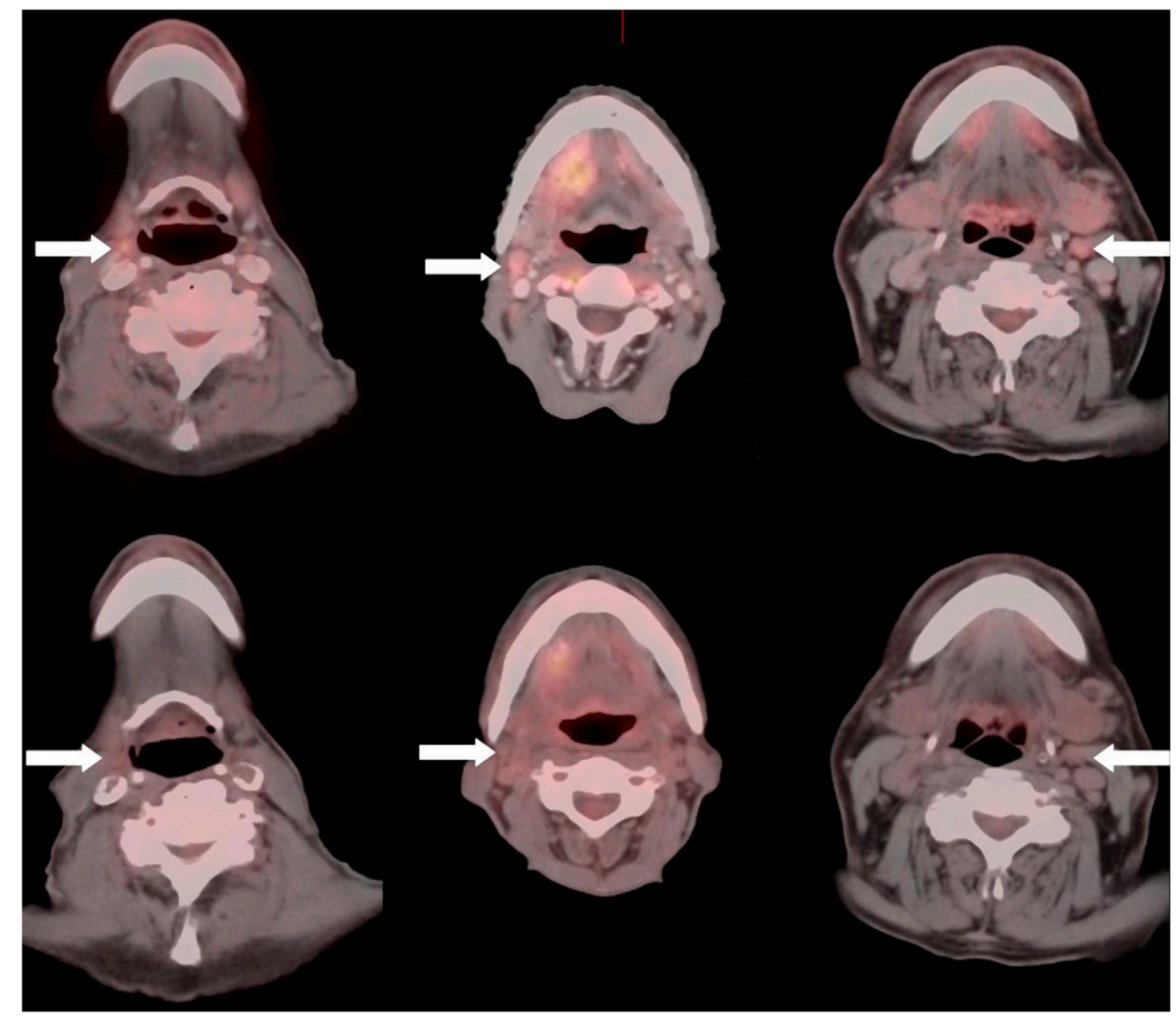

FIGURE 7. Three examples in which HN PET/CT protocol (upper panel) detected small ( $<15 \mathrm{~mm}$ ) histologically proven positive lymph nodes that were missed by standard WB protocol (lower panel). Arrows indicate discordant nodes.

$(13,16)$, the change in management is often due to incidental findings unrelated to the primary $\mathrm{HN}$ tumor. In the current study, synchronous unsuspected primary tumors were identified in $7 \%$ ( 3 of 44 patients), which is similar to the reported rate of $4 \%-8 \%(11,13,29)$. These tumors included a colon cancer, a lymphoma, and metastatic melanoma. Although significant, these were, nonetheless, incidental findings.

\section{CONCLUSION}

Both WB and HN PET/CT outperform CECT in the identification of the primary HN tumor. The WB PET/CT protocol offers no significant advantage over a highly dedicated, high-quality CECT alone in the detection of nodal metastasis, either on a per-level or on a per-patient basis. The
HN PET/CT protocol demonstrates a significantly better performance than both the CECT and the WB PET/CT protocols in detecting nodal metastases on a per-level basis and a near-significant improvement over WB PET/CT on a perpatient basis. The primary advantage of the HN PET/CT protocol over the WB PET/CT protocol is in the detection of small $(<15 \mathrm{~mm})$ nodal metastasis, in which a 4-fold increase in nodal detection is found by the HN versus the WB PET/CT protocol.

\section{ACKNOWLEDGMENT}

This work was supported in part by a Coordenação de Aperfeiçoamento de Pessoal de Nível Superior (CAPES) fellowship and a Conselho Nacional de Pesquisa (CNPq) fellowship. 


\section{REFERENCES}

1. Schoder H, Yeung HW, Gonen M, Kraus D, Larson SM. Head and neck cancer: clinical usefulness and accuracy of PET/CT image fusion. Radiology. 2004; 231:65-72.

2. Di Martino E, Nowak B, Hassan HA, et al. Diagnosis and staging of head and neck cancer: a comparison of modern imaging modalities (positron emission tomography, computed tomography, color-coded duplex sonography) with panendoscopic and histopathologic findings. Arch Otolaryngol Head Neck Surg. 2000;126: 1457-1461.

3. Kitagawa Y, Nishizawa S, Sano K, et al. Whole-body ${ }^{18}$ F-fluorodeoxyglucose positron emission tomography in patients with head and neck cancer. Oral Surg Oral Med Oral Pathol Oral Radiol Endod. 2002;93:202-207.

4. Goerres GW, Schmid DT, Gratz KW, von Schulthess GK, Eyrich GK. Impact of whole body positron emission tomography on initial staging and therapy in patients with squamous cell carcinoma of the oral cavity. Oral Oncol. 2003; 39:547-551.

5. McCollum AD, Burrell SC, Haddad RI, et al. Positron emission tomography with ${ }^{18} \mathrm{~F}$-fluorodeoxyglucose to predict pathologic response after induction chemotherapy and definitive chemoradiotherapy in head and neck cancer. Head Neck. 2004;26:890-896.

6. Goerres GW, Schmid DT, Bandhauer F, et al. Positron emission tomography in the early follow-up of advanced head and neck cancer. Arch Otolaryngol Head Neck Surg. 2004;130:105-109.

7. Goshen E, Yahalom R, Talmi YP, Rotenberg G, Oksman Y, Zwas ST. The role of gamma-PET in the evaluation of patients with recurrent squamous cell cancer of the head and neck. Int J Oral Maxillofac Surg. 2005;34:386-390.

8. Schwartz DL, Rajendran J, Yueh B, et al. FDG-PET prediction of head and neck squamous cell cancer outcomes. Arch Otolaryngol Head Neck Surg. 2004;130: 1361-1367.

9. Agarwal V, Branstetter BF IV, Johnson JT. Indications for PET/CT in the head and neck. Otolaryngol Clin North Am. 2008;41:23-49.

10. Sigg MB, Steinert H, Gratz K, Hugenin P, Stoeckli S, Eyrich GK. Staging of head and neck tumors: $\left[{ }^{18} \mathrm{~F}\right]$ fluorodeoxyglucose positron emission tomography compared with physical examination and conventional imaging modalities. J Oral Maxillofac Surg. 2003;61:1022-1029.

11. Ng SH, Yen TC, Liao CT, et al. ${ }^{18}$ F-FDG PET and CT/MRI in oral cavity squamous cell carcinoma: a prospective study of 124 patients with histologic correlation. J Nucl Med. 2005;46:1136-1143.

12. Kim SY, Roh J-L, Yeo N-K, et al. Combined ${ }^{18}$ F-fluorodeoxyglucose-positron emission tomography and computed tomography as a primary screening method for detecting second primary cancers and distant metastases in patients with head and neck cancer. Ann Oncol. 2007;18:1698-1703.

13. Branstetter BF IV, Blodgett TM, Zimmer LA, et al. Head and neck malignancy: is PET/CT more accurate than PET or CT alone? Radiology. 2005;235:580-586.

14. Fleming AJ Jr, Smith SP Jr, Paul CM, et al. Impact of $\left[{ }^{18} \mathrm{~F}\right]$-2-fluorodeoxyglucose-positron emission tomography/computed tomography on previously untreated head and neck cancer patients. Laryngoscope. 2007;117:1173-1179.
15. Gordin A, Daitzchman M, Doweck I, et al. Fluorodeoxyglucose-positron emission tomography/computed tomography imaging in patients with carcinoma of the larynx: diagnostic accuracy and impact on clinical management. Laryngoscope. 2006;116:273-278.

16. Gordin A, Golz A, Keidar Z, Daitzchman M, Bar-Shalom R, Israel O. The role of FDG-PET/CT imaging in head and neck malignant conditions: impact on diagnostic accuracy and patient care. Otolaryngol Head Neck Surg. 2007;137: $130-137$.

17. Scott AM, Gunawardana DH, Bartholomeusz D, Ramshaw JE, Lin P. PET changes management and improves prognostic stratification in patients with head and neck cancer: results of a multicenter prospective study. J Nucl Med. 2008; 49:1593-1600.

18. Yamamoto Y, Wong TZ, Turkington TG, Hawk TC, Coleman RE. Head and neck cancer: dedicated FDG PET/CT protocol for detection-phantom and initial clinical studies. Radiology. 2007;244:263-272.

19. Hain SF. Positron emission tomography in cancer of the head and neck. $\mathrm{Br} \mathrm{J}$ Oral Maxillofac Surg. 2005;43:1-6.

20. De Bondt RB, Nelemans PJ, Hofman PA, et al. Detection of lymph node metastases in head and neck cancer: a meta-analysis comparing US, USgFNAC, CT and MR imaging. Eur J Radiol. 2007;64:266-272.

21. Lan XL, Zhang YX, Wu ZJ, Jia Q, Wei H, Gao ZR. The value of dual time point ${ }^{18}$ F-FDG PET imaging for the differentiation between malignant and benign lesions. Clin Radiol. 2008;63:756-764.

22. Zytoon AA, Murakami K, El-Kholy MR, El-Shorbagy E. Dual time point FDGPET/CT imaging: potential tool for diagnosis of breast cancer. Clin Radiol. 2008;63:1213-1227.

23. Hustinx R, Smith RJ, Benard F, et al. Dual time point fluorine-18 fluorodeoxyglucose positron emission tomography: a potential method to differentiate malignancy from inflammation and normal tissue in the head and neck. Eur $J$ Nucl Med. 1999;26:1345-1348.

24. Zhuang H, Pourdehnad M, Lambright ES, et al. Dual time point ${ }^{18}$ F-FDG PET imaging for differentiating malignant from inflammatory processes. J Nucl Med. 2001;42:1412-1417.

25. Veit-Haibach P, Luczak C, Wanke I, et al. TNM staging with FDG-PET/CT in patients with primary head and neck cancer. Eur J Nucl Med Mol Imaging. 2007;34:1953-1962.

26. AAssar OS, Fischbein NJ, Caputo GR, et al. Metastatic head and neck cancer: role and usefulness of FDG PET in locating occult primary tumors. Radiology. 1999;210:177-181.

27. Ekberg T, Sörensen J, Engström M, Blomquist E, Sundin A, Anniko M. Clinical impact of positron emission tomography (PET) with $\left({ }^{18} \mathrm{~F}\right)$ fluorodeoxyglucose (FDG) in head and neck tumours. Acta Otolaryngol. 2007;127:186-193.

28. Nabili V, Zaia B, Blackwell KE, Head CS, Grabski K, Sercarz JA. Positron emission tomography: poor sensitivity for occult tonsillar cancer. Am J Otolaryngol. 2007;28:153-157.

29. Ishimori T, Patel PV, Wahl RL. Detection of unexpected additional primary malignancies with PET/CT. J Nucl Med. 2005;46:752-757. 